\title{
Non-insulin determinant pathways maintain glucose homeostasis upon metabolic surgery
}

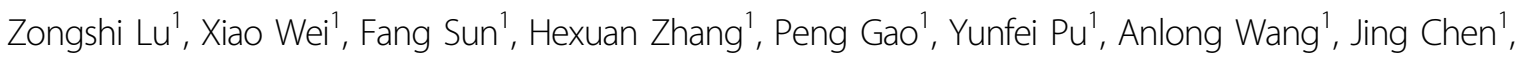 \\ Weidong Tong ${ }^{2}$, Qiang Li', Xunmei Zhou', Zhencheng Yan' ${ }^{1}$, Hongting Zheng $\mathbb{B}^{3}$, Gangyi Yang $\mathbb{1}^{4}$, Yu Huang ${ }^{5}$, \\ Daoyan Liu ${ }^{1}$ and Zhiming Zhu ${ }^{1}$
}

\begin{abstract}
Insulin is critical for glucose homeostasis, and insulin deficiency or resistance leads to the development of diabetes. Recent evidence suggests that diabetes can be remitted independent of insulin. However, the underlying mechanism remains largely elusive. In this study, we utilized metabolic surgery as a tool to identify the non-insulin determinant mechanism. Here, we report that the most common metabolic surgery, Roux-en-Y gastric bypass (RYGB), reduced insulin production but persistently maintained euglycemia in healthy Sprague-Dawley (SD) rats and C57 mice. This reduction in insulin production was associated with RYGB-mediated inhibition of pancreatic preproinsulin and polypyrimidine tract-binding protein 1. In addition, RYGB also weakened insulin sensitivity that was evaluated by hyperinsulinemic-euglycemic clamp test and downregulated signaling pathways in insulin-sensitive tissues. The mechanistic evidence suggests that RYGB predominately shifted the metabolic profile from glucose utilization to fatty acid oxidation, enhanced the energy expenditure and activated multiple metabolic pathways through reducing gut energy uptake. Importantly, the unique effect of RYGB was extended to rats with islet disruption and patients with type 2 diabetes. These results demonstrate that compulsory rearrangement of the gastrointestinal tract can initiate non-insulin determinant pathways to maintain glucose homeostasis. Based on the principle of RYGB action, the development of a noninvasive intervention of the gastrointestinal tract is a promising therapeutic route to combat disorders characterized by energy metabolism dysregulation.
\end{abstract}

\section{Introduction}

Insulin has been deemed the sole hypoglycemic hormone in mammals since its discovery in $1921^{1}$. A generally accepted concept is that insulin is essential for maintaining glucose homeostasis and regulating carbohydrate, lipid, and protein metabolism through eliciting a diverse array of signaling pathways by binding to its

\footnotetext{
Correspondence: Zhiming Zhu (zhuzm@yahoo.com)

${ }^{1}$ Department of Hypertension and Endocrinology, Center for Hypertension and Metabolic Diseases, Daping Hospital, Third Military Medical University,

Chongqing Institute of Hypertension, Chongqing 400042, China

${ }^{2}$ Department of Gastrointestinal Metabolic Surgery, Daping Hospital, Third

Military Medical University, Chongqing 400042, China

Full list of author information is available at the end of the article.

These authors contributed equally: Zongshi Lu Xiao Wei, Fang Sun
}

specific receptor ${ }^{2-4}$. The pathogenesis of diabetes is characterized by the progressive development of insulin resistance and a deficiency in insulin secretion, leading to overt hyperglycemia ${ }^{3-5}$. Therefore, current diabetes therapies focus on insulin replenishment and insulin sensitivity improvement through medicine and lifestyle changes ${ }^{5}$. Outside of classical management strategies, metabolic surgery is presently thought to be the most durable intervention for the remission of diabetes and obesity $^{4}$. Roux-en-Y gastric bypass (RYGB) is the most effective and widely performed metabolic surgery ${ }^{6}$. RYGB can quickly correct hyperglycemia and hyperinsulinemia, despite the presence of continued insulin resistance and obesity in patients with type 2 diabetes $^{7}$. In addition to

\section{(c) The Author(s) 2018}

(c) (i) Open Access This article is licensed under a Creative Commons Attribution 4.0 International License, which permits use, sharing, adaptation, distribution and reproduction cc) in any medium or format, as long as you give appropriate credit to the original author(s) and the source, provide a link to the Creative Commons license, and indicate if changes were made. The images or other third party material in this article are included in the article's Creative Commons license, unless indicated otherwise in a credit line to the material. If material is not included in the article's Creative Commons license and your intended use is not permitted by statutory regulation or exceeds the permitted use, you will need to obtain permission directly from the copyright holder. To view a copy of this license, visit http://creativecommons.org/licenses/by/4.0/. 
weight loss and glycemic control, metabolic surgery also improves the micro- and macrovascular complications of diabetes and even of cancers ${ }^{8,9}$. Although metabolic surgery-mediated changes in gut hormones, nutrient sensing, intestinal glucose utilization, microbiota, and bile acid metabolism in diabetes and obesity have been proposed $^{10-14}$, the underlying mechanism remains elusive. Furthermore, several studies suggest that central leptin administration and RYGB are sufficient to restore euglycemia in the insulin-deficient state, which clearly indicates the existence of an insulin-independent hypoglycemic phenomenon, but its underlying mechanism is poorly understood ${ }^{15,16}$. An unsolved question is how glucose homeostasis can be maintained without the participation of insulin. One important obstacle to addressing this issue is that most studies investigate the animal models or patients with diabetes or obesity, which is unable to exclude the involvement of insulin action $^{4,5,17}$. Inconceivably, little is known about how metabolic surgery affects islet function and glucose homeostasis under physiological conditions. This lack of knowledge tremendously impedes our understanding of mechanisms related to metabolic surgery. To rectify this limitation, we studied the effect of metabolic surgery on glucose homeostasis by eliminating insulin sensitivity and by chemically disrupting islets in healthy rodents. We hypothesized that gastrointestinal rearrangement by metabolic surgery might initiate unique pathways to maintain glucose homeostasis. Thus, we applied RYGB surgery as a tool to study how gastrointestinal intervention affects insulin production and action and the maintenance of glucose homeostasis in a physiological setting, and further validated the findings in the diabetic state.

\section{Results \\ RYGB reduces insulin production but maintains euglycemia}

First, we examined how metabolic surgery affects islet function under physiological conditions. RYGB surgery, which connects a small stomach pouch to the midjejunum and bypasses the entire stomach and duodenum, was performed in this study (Fig. 1a). To avoid the interference of insulin defects or resistance, healthy Sprague-Dawley (SD) rats were examined in this study. For dynamic probing of changes in blood glucose levels, we used an updated ambulatory glucose monitoring technique for rats ${ }^{18}$. RYGB predominately reduced basal and glucose-stimulated insulin levels in plasma (Fig. 1b, Supplementary Fig. S1a), but maintained euglycemia in rats (Fig. 1c, d). However, RYGB did not change islet histology; rather, reductions in body weight, food and water intake and urine output were observed in the rats
(Fig. 1e and Supplementary Fig. S1b-e). Next, we asked how RYGB reduces plasma insulin levels. Preproinsulin and proinsulin are the precursors of insulin ${ }^{19,20}$. RYGB reduced both pancreatic preproinsulin mRNA and plasma proinsulin levels in rats (Fig. 1f, g). Polypyrimidine tractbinding protein 1 (PTBP1) encodes an insulin granule protein and promotes insulin translation and stabilization $^{21}$. We showed that RYGB remarkably reduced PTBP1 expression at both the mRNA and protein levels (Fig. 1i). Accordingly, RYGB also decreased glucose-induced insulin secretion, as depicted by the area under the curve (AUC) (Fig. 1j). These results suggest that RYGB surgery reduces insulin production and secretion in intact islet conditions but still maintains glucose homeostasis.

\section{The RYGB-mediated improvement in glucose homeostasis is independent of insulin sensitivity}

Next, we asked whether RYGB affects insulin sensitivity and its related signaling and metabolic pathways. RYGB did not alter oral glucose tolerance (OGT) but did enhance intraperitoneal glucose tolerance (IPGT) after high-concentration glucose infusion in rats (Fig. 2a-c). In this study, we evaluated insulin sensitivity using the hyperinsulinemic-euglycemic clamp test in conscious rats. Insulin is the dominant repressor of hepatic glucose production $(\mathrm{HGP})^{22,23}$. Interestingly, RYGB reduced insulin sensitivity in conscious rats and enhanced HGP under both basal and hyperinsulinemic-euglycemic conditions (Fig. $2 \mathrm{~d}-\mathrm{f}$ ), indicating that RYGB attenuated the inhibitory effect of insulin on HGP. In addition, hepatic gluconeogenesis is catalyzed by several key rate-limiting enzymes, such as phosphoenol pyruvate carboxykinase (PEPCK) and glucose-6-phosphatase (G6Pase) ${ }^{24,25}$. Consistent with the enhanced HGP, hepatic PEPCK and G6Pase expression was upregulated under high insulin clamp conditions (Fig. 2f, g). Furthermore, glucose uptake into muscle and adipose tissue was significantly decreased in RYGB rats compared with sham rats, suggesting reduced insulin activity in these insulin-sensitive tissues (Fig. 2h). The glucose uptake by brain or jejunum was increased, whereas it was decreased in heart and not significantly changed in liver and kidney (Supplementary Fig. S2a). Importantly, phosphorylation of the insulin receptor in skeletal muscle was lower in RYGB rats than in sham rats (Fig. 2i). RYGB elevated glucagon-like peptide 1 (GLP-1) levels and decreased dipeptidyl peptidase 4 (DPP-4) and leptin levels in rats without affecting the plasma levels of other gut hormones and adipokines (Supplementary Fig. S2b-i). Taken together, the results indicate that the effect of RYGB on glucose homeostasis is independent of insulin sensitivity; RYGB antagonizes insulin action under physiological conditions. 


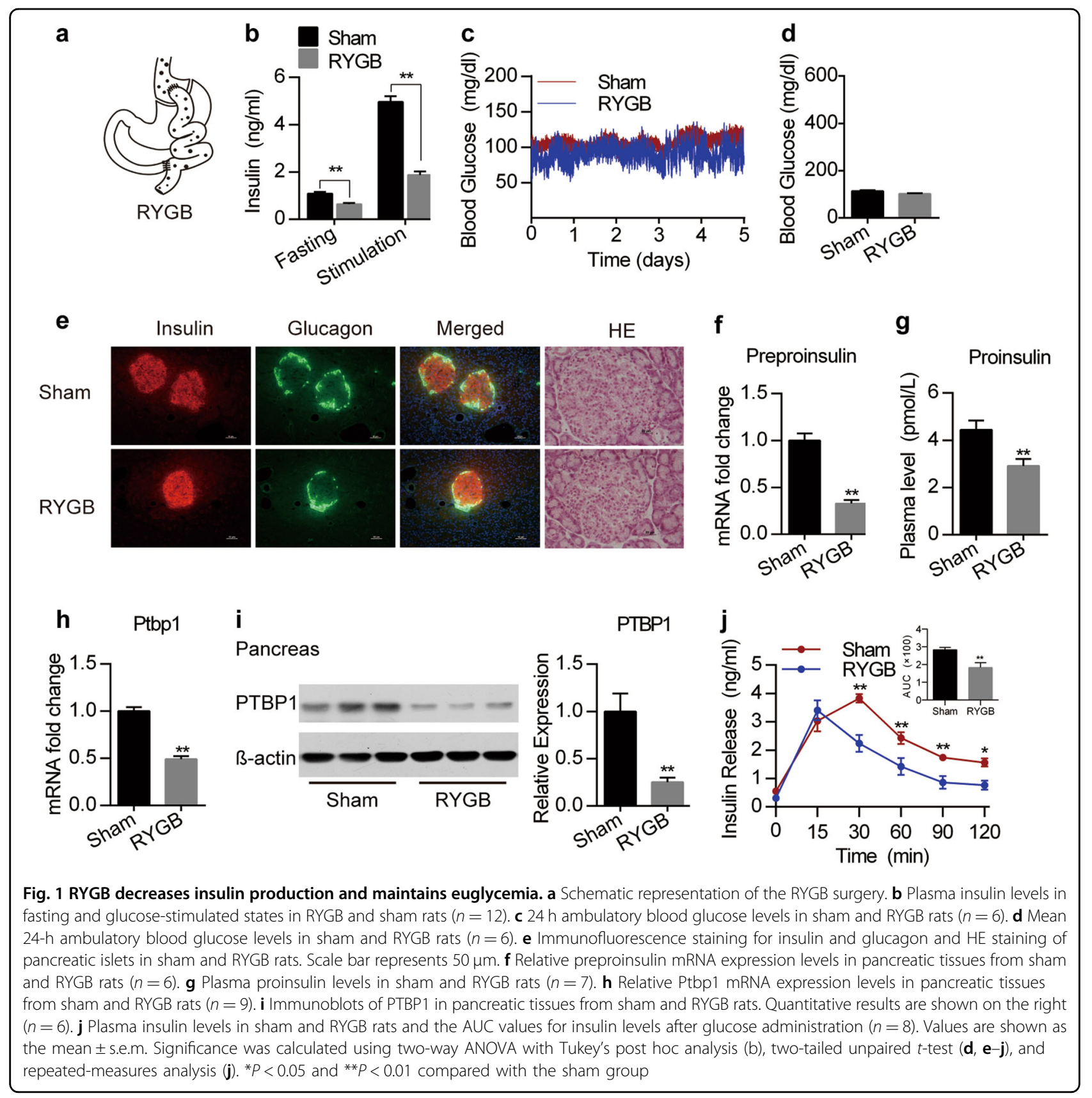

Eliminating the stomach and duodenum by RYGB reduces insulin production and restricts energy uptake

We further asked which part of the gastrointestinal tract affects glucose homeostasis. We compared the effects of three metabolic surgeries that transform the gastrointestinal tract anatomy in different manners (Fig. 3a). The RYGB procedure is shown in Fig. 1a; the duodenaljejunal bypass (DJB) procedure bypasses the duodenum and proximal jejunum, while vertical sleeve gastrectomy (VSG) excises most of the stomach ${ }^{26}$. In some rats subjected to RYGB, the original alimentary tract was restored to further confirm the effects of RYGB (Fig. 3a). RYGB, not the other surgical procedures, persistently prevented weight gain and reduced food intake. However, these effects were reversed by alimentary tract restoration (Fig. 3b and d). Importantly, among these surgical procedures, only RYGB significantly reduced plasma insulin levels in rats (Fig. 3c), indicating that the intact stomach and duodenum are critical for insulin production. The intraperitoneal glucose tolerance test (IPGTT), oral glucose tolerance test (OGTT) and intraperitoneal insulin tolerance test (IPITT) results were not affected by the different surgeries (Supplementary Fig. S3a-f). Nutrient digestion and absorption dramatically affect insulin 


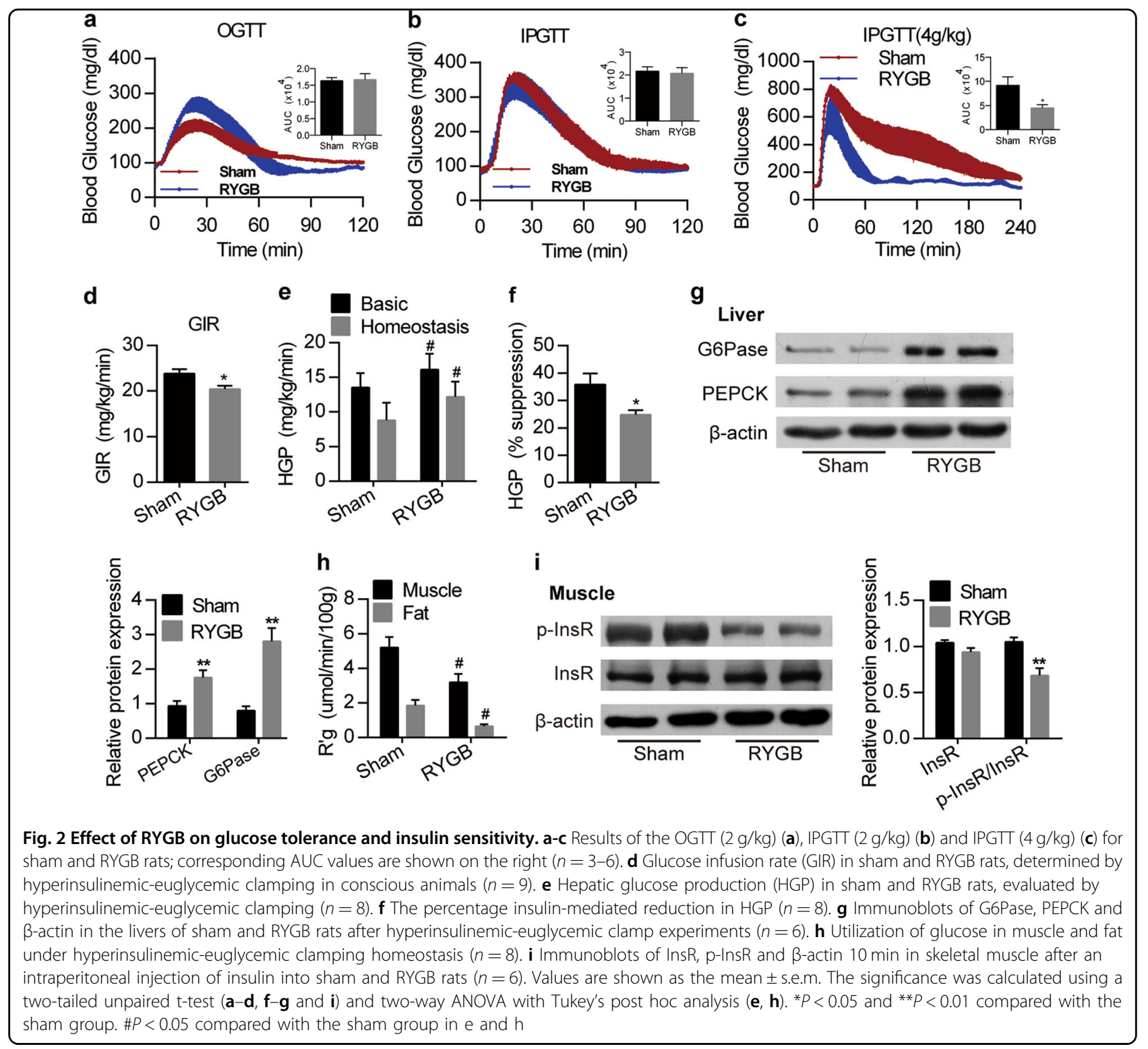

synthesis through nutrient sensing ${ }^{27,28}$. Indeed, analysis of fecal components revealed that RYGB, not the other surgical procedures, reduced energy uptake derived from nutrient absorption by approximately 37\% (Fig. 3e). However, none of these surgical procedures altered the proportion of absorbed nutrients in rats (Fig. 3f). We further performed caloric restriction (CR) in rats, whose food intake was $60 \%$ of that in sham group. Plasma insulin level was decreased in CR group compared with sham group, but still higher than RYGB group (Supplementary Fig. S3g-i). These results indicate that stomach and duodenum bypass plays a crucial role in the regulation of insulin production through controlling gut energy uptake.
RYGB shifts the metabolic profile to maintain glucose homeostasis

Then, we asked how RYGB surgery maintains glucose homeostasis in an insulin-paucity state. We showed a clear shift in energy substrate usage from glucose to fatty acids in rodents, as evidenced by a reduced respiratory exchange ratio (RER) at day and night and by increased energy expenditure (EE) in RYGB mice (Fig. 4a-e), suggesting the increasing utilization of glucose and fatty acids as the main energy substrates. Accordingly, octanoyl-fueled mitochondrial oxygen consumption, representing fatty acid oxidation (FAO) in mitochondria, increased in the liver and skeletal muscle 


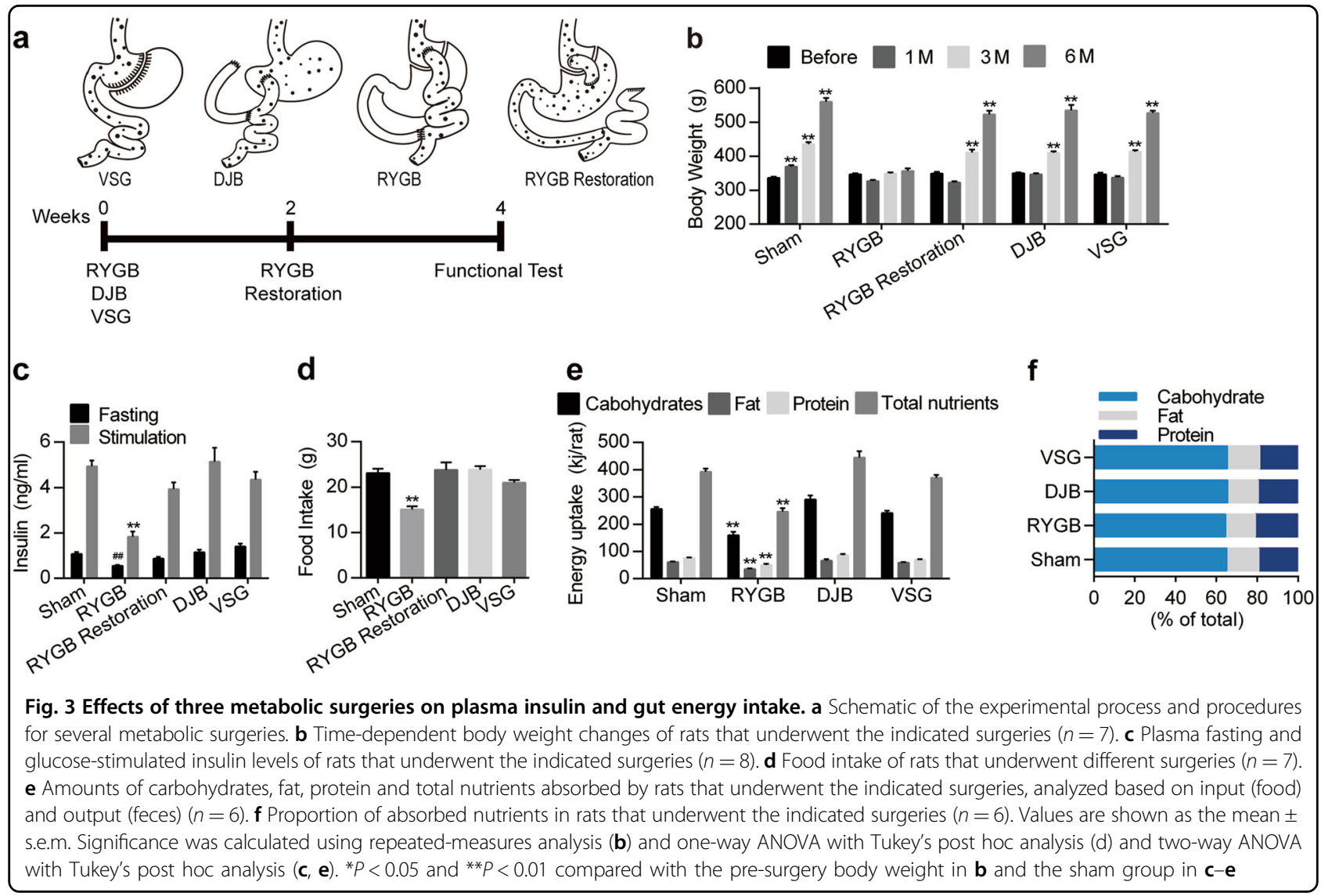

in RYGB rats (Fig. $4 \mathrm{f}-\mathrm{g})^{29}$. To further explore the metabolic changes after surgery, we performed mRNA microarray experiments on liver, skeletal muscle and adipose tissue. Significantly changed genes (fold change $>2$ ) were identified to perform KEGG pathway analysis (Fig. 4h). In the liver, metabolic-related pathways, such as fatty acid metabolism (including degradation and biosynthesis), glucose metabolism (including pyruvate metabolism and glycolysis/gluconeogenesis) and metabolism-related signaling pathways (including AMPK, peroxisome proliferator activated receptors (PPARs), and adipocytokine), were significantly upregulated. However, the phosphatidylinositol-3 kinase (PI3K)-Akt signaling pathway was downregulated, which corresponded with the result of insulin insensitivity detected by the hyperinsulinemic-euglycemic clamp test in vivo after surgery (Fig. $5 \mathrm{f}-\mathrm{g}$ ). Similarly, PPAR upregulation and PI3K-Akt downregulation were found in skeletal muscle and adipose tissue (Supplementary Fig. S4a, b, Supplementary Fig. S5b-e). Collectively, these results demonstrate that gastrointestinal tract rearrangement alters the manner of energy utilization and activates multiple glucose and fatty acid metabolism-related signaling pathways under insulinpaucity conditions.

\section{RYGB alters glucose- and fatty acid-related metabolic targets}

To verify the mRNA microarray chip results in vivo, some differentially expressed metabolism-associated genes are shown in the heatmap (Fig. 5a, Supplementary Fig. S5a). The enriched genes included key enzymes involved in glucose and fatty acid metabolism. We confirmed the changes in the expression of some of these genes in liver, skeletal muscle and adipose tissue using western blotting. In the rat liver, key genes in glycolysis were enhanced, including glucokinase (GCK), phosphofructokinase liver type (PFKL), and pyruvate kinase isozymes R/L (PKLR). For pyruvate metabolism, pyruvate dehydrogenase kinase (PDHK1) was downregulated (Fig. 5b, c). Regarding fatty acid metabolism, the phosphorylation of acetyl-CoA carboxylase (ACC) and the expression of the FAO key enzyme carnitine palmitoyltransferase-1 (CPT1) were enhanced to promote FAO (Fig. 5d, e). In skeletal muscle, genes involved in glycolysis, such as hexokinase 2 (HK2), 6phosphofructokinase 1 (PFK1) and pyruvate kinase muscle isozyme 1/2 (PKM1/2), were upregulated after RYGB (Supplementary Fig. S5b, c). In adipose tissue, CPT1 increased after RYGB (Supplementary Fig. S5d, e). These results suggest that RYGB triggers complex changes in the 


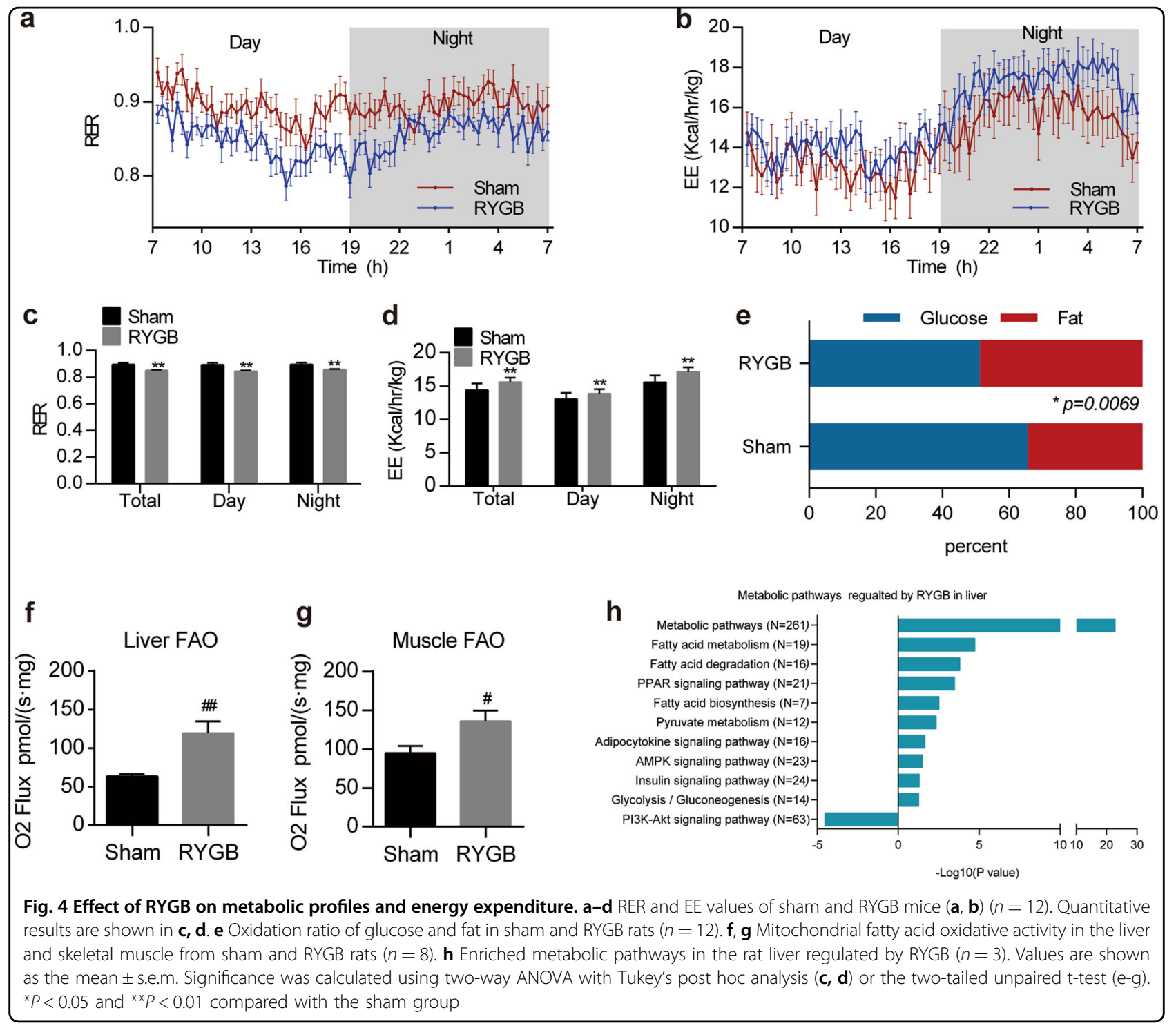

metabolic networks related to glucose and fatty acid metabolism rather than alterations in a single gene or pathway.

\section{The effect of RYGB on glucose homeostasis has been validated in islet-disrupted rats}

We have demonstrated that RYGB can maintain glucose homeostasis in an insulin-paucity state under physiological conditions. We reasoned that this effect can be validated during insulin deficiency. Thus, we disrupted rat islets through a single bolus peritoneal injection of streptozocin (STZ). STZ administration severely destroyed islets (Fig. 6a) and tremendously decreased plasma insulin levels in SD rats (Fig. 6c). STZ-treated rats displayed typical features of diabetes (Supplementary Fig. S6a-d). However, RYGB restored robust hypergly- cemia to euglycemia within one week in STZ-treated rats compared with sham surgery (Fig. 6b). Furthermore, the insulin-deficient state was not altered in STZ-treated rats before and after surgery (Fig. 6c). To further examine the changes in blood glucose, a glucose telemetric transmitter was surgically implanted into the distal portion of the descending aorta. The results further validated that RYGB persistently maintained long-term euglycemia in rats with islet disruption (Fig. 6d, e). In addition, IPGT and OGT were significantly improved in STZ-treated rats after surgery (Fig. 6f-h). However, insulin sensitivity, which was evaluated by IPITT, was declined in STZ-treated rats after surgery (Fig. 6i). These results indicate that RYGB can rapidly antagonize hyperglycemia and maintain glucose homeostasis, even in conditions of severe islet disruption. 
a
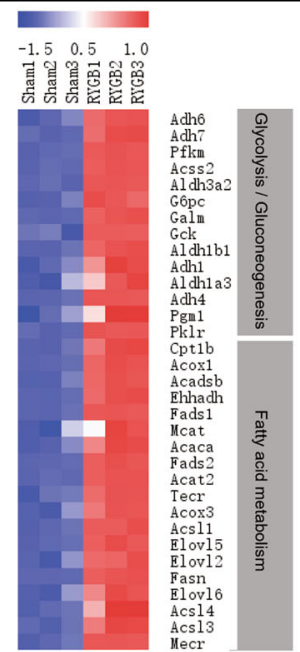

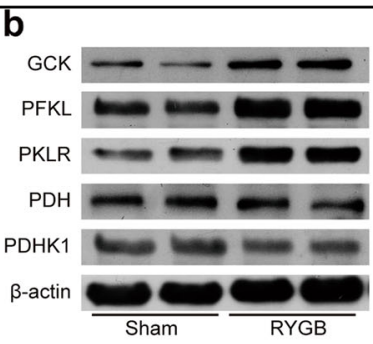

\section{C}

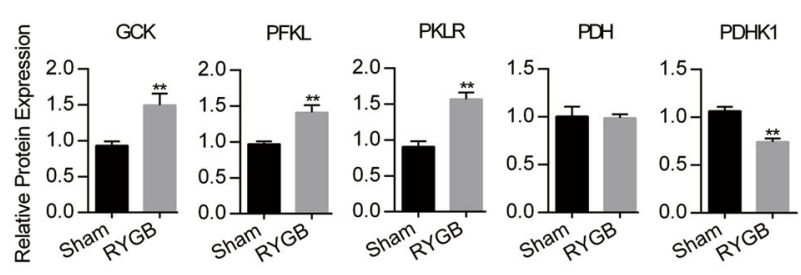

d
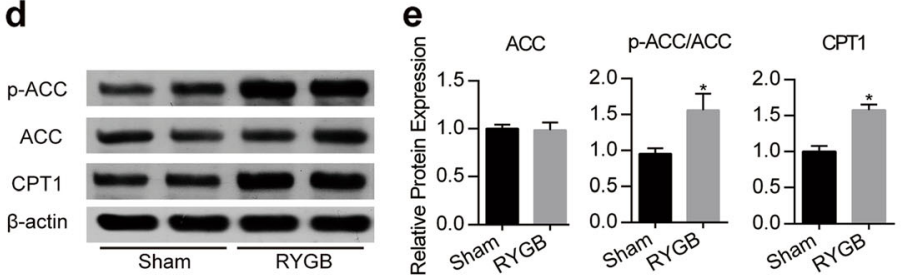

g
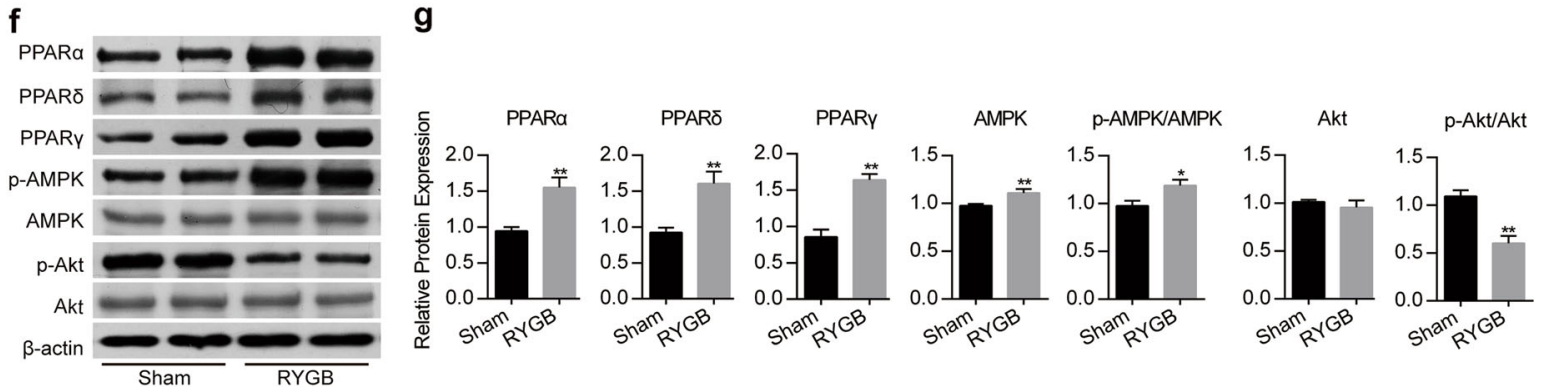

Fig. 5 Changes in metabolic-associated genes and enzymes in the liver. a Expression levels of enriched glycolysis/gluconeogenesis and fatty acid metabolism pathway genes in the livers of sham and RYGB rats $(n=3)$. $\mathbf{b}, \mathbf{c}$ Immunoblots of GCK, PFKL, PKLR, PDH, PDHK1 and $\beta$-actin in the liver from sham and RYGB rats $(n=6)$. $\mathbf{d}$, e Immunoblots of p-ACC, t-ACC, CPT1 and $\beta$-actin in the liver from sham and RYGB rats $(n=6)$. $\mathbf{f}, \mathbf{g}$ Immunoblots of PPARa, PPARß, PPARS, p-AMPK, AMPK, p-Akt, Akt and $\beta$-actin in the liver from sham and RYGB rats $(n=6)$. Values are shown as the mean \pm s.e.m. Significance was calculated using a two-tailed unpaired t-test ( $\mathbf{c}$, e and $\mathbf{g}$ ). ${ }^{*} P<0.05$ and ${ }^{* *} P<0.01$ compared with the sham group

The ability of RYGB to remit hyperglycemia is independent of islet state in patients with type 2 diabetes

Currently, RYGB is not recommended for patients with type 2 diabetes with severe islet lesions ${ }^{6}$. However, the beneficial effect of RYGB in patients with type 1 diabetes has also been reported ${ }^{30}$. In this study, we examined the relationship between islet function and blood glucose change in patients with type 2 diabetes before and after RYGB. General characteristics of the patients are included in the supplemental information (Supplementary Table S1). We showed that the average ambulatory and fasting blood glucose levels were significantly reduced in patients with diabetes after surgery (Fig. 7a, b). Compared with the OGT test results before surgery, those for patients with diabetes after surgery were improved and maintained over long term (Fig. 7c, d). RYGB led to a persistent decline in $\mathrm{HbA} 1 \mathrm{c}$ and body mass index (BMI) in patients with diabetes compared with the pre-surgery values (Fig. 7e). RYGB decreased the plasma levels of proinsulin, insulin and $\mathrm{C}$-peptide in patients with diabetes (Fig. $7 \mathrm{f}-\mathrm{h}$ ). Currently, most clinical studies have shown that RYGB decreases plasma insulin levels in obese patients with type 2 diabetes $^{31-33}$. Our clinical evidence further indicates that the euglycemic status maintained by RYGB might be independent of insulin and islet state in patients with diabetes.

\section{Discussion}

In this study, we utilized RYGB metabolic surgery as a tool to identify the non-insulin determinate mechanism that is crucial for maintaining glucose homeostasis. Importantly, we reveal that gastrointestinal tract rearrangement by RYGB reduces both insulin production and action but persistently maintains euglycemia in healthy rats. The mechanistic evidence suggests that RYGB antagonizes pancreatic preproinsulin expression and the insulin signaling pathway in several insulin-sensitive tissues. Due to the restriction of gut energy uptake, RYGB predominately shifts the metabolic profile from glucose utilization to FAO, enhances EE and triggers multiple metabolic pathways. Importantly, these unique effects of RYGB can be extended to rats with islet disruption and 

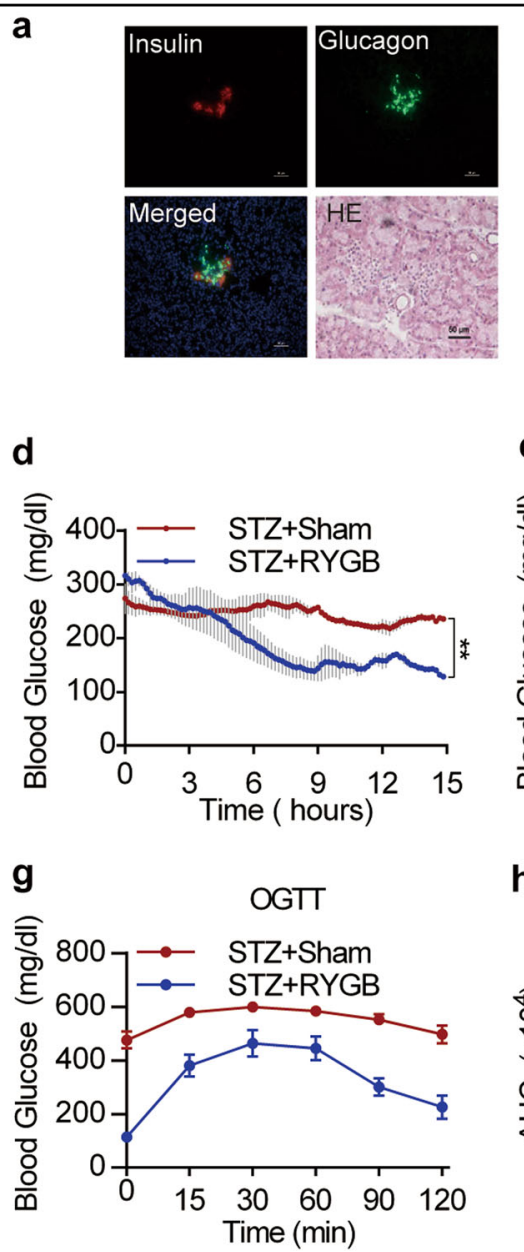
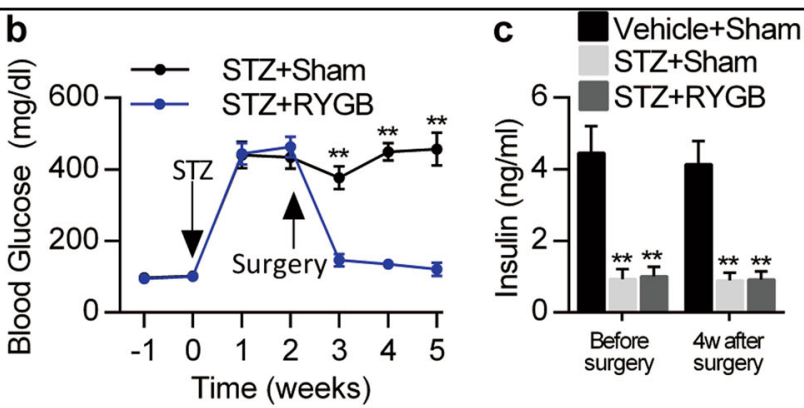

e
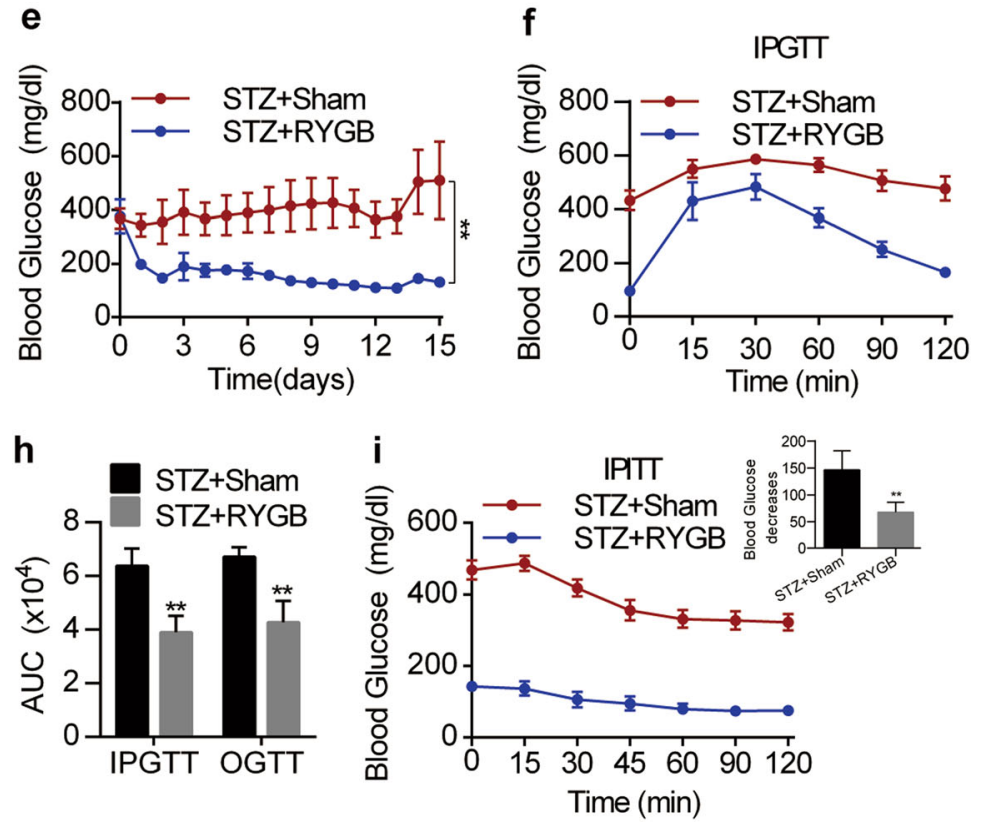

Fig. 6 Effect of RYGB on glucose homeostasis in islet-disrupted rats. a Immunofluorescence staining for insulin and glucagon and HE staining of pancreatic islets in STZ-treated rats. Scale bar represents $50 \mu \mathrm{m}$. b Weekly fasting blood glucose levels of the indicated groups from -1 to 5 weeks $(n$ $=7)$. c Plasma insulin levels in sham or RYGB treated vehicle or STZ injected rats before and 4 weeks after surgery $(n=7)$. $\mathbf{d}$ Ambulatory blood glucose levels in sham- or RYGB-treated STZ injected rats within $15 \mathrm{~h}$ after surgery $(n=2)$. e Mean 24-h ambulatory blood glucose levels sham- or RYGB-treated STZ injected rats within 15 days after surgery $(n=2)$. $\mathbf{f}-\mathbf{h}$ Results of the IPGTT ( $\mathbf{f})$ and OGTT $(\mathbf{g})$ for sham- or RYGB-treated STZ injected rats; corresponding AUC values are shown in $\mathrm{H}(n=4-5)$. $\mathbf{i}$ IPITT for sham- or RYGB-treated STZ injected rats. Values are shown as the mean \pm s.e.m. Significance was calculated using repeated-measures analysis (b) and two-way ANOVA with Tukey's post hoc analysis (c-e), and two-tailed unpaired t-test $(\mathbf{h}$ and $\mathbf{i}){ }^{*} P<0.05$ and ${ }^{*} P<0.01$ compared with the surgery group in $\mathbf{b}$ and $\mathbf{d}-\mathbf{e}$, compared with the sham group in $\mathbf{h}$ and compared with the vehicle + sham group in $\mathbf{c}$

patients with type 2 diabetes. Based on our experimental and clinical results, we propose that excluding the stomach and duodenum by RYGB in rats largely reduces energy intake, which decreases insulin production and triggers insulin-independent metabolic pathways. Consequently, glucose homeostasis is maintained by noninsulin-dependent mechanisms (Fig. 8).

The liver, skeletal muscle and fat harmonize to maintain whole-body glucose homeostasis ${ }^{3}$. Ingested glucose is absorbed from the gastrointestinal tract into the portal vein and liver. There, the liver eliminates and stores a large fraction of ingested glucose while decreasing the amount of hepatic glucose entering the circulation ${ }^{34}$. Insulin is a depressor of HGP, whereas hepatic glucose uptake is less sensitive to insulin ${ }^{22}$. Conversely, when glucose is no longer absorbed from the gastrointestinal tract, HGP will increase to avoid hypoglycemia through declining insulin secretion from pancreatic islets. In this study, RYGB resulted in a $37 \%$ reduction of ingested nutrients by eliminating the stomach and duodenum. This compulsory restriction of gut energy uptake inhibits insulin synthesis because starvation or caloric restriction significantly decreases plasma insulin levels in humans ${ }^{35}$. In addition, insulin is derived from preproinsulin and proinsulin ${ }^{19,20}$. PTBP1 binds mRNAs that encode insulin granule proteins and promotes insulin translation and stabilization ${ }^{21}$. We found that RYGB directly inhibited pancreatic preproinsulin and PTBP1 expression and 


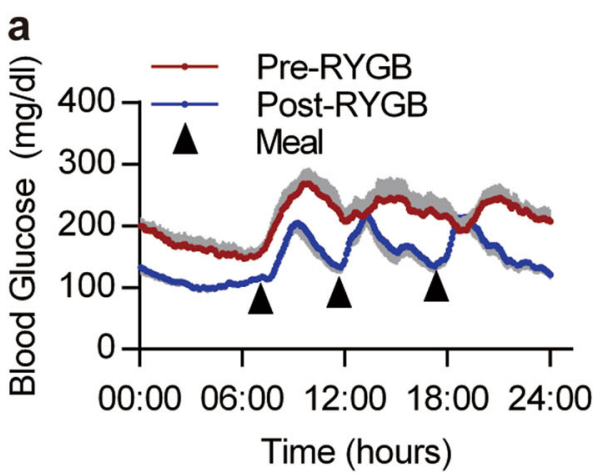

d

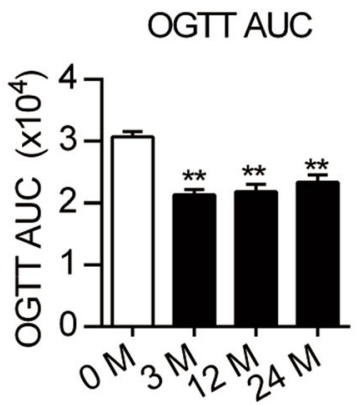

e

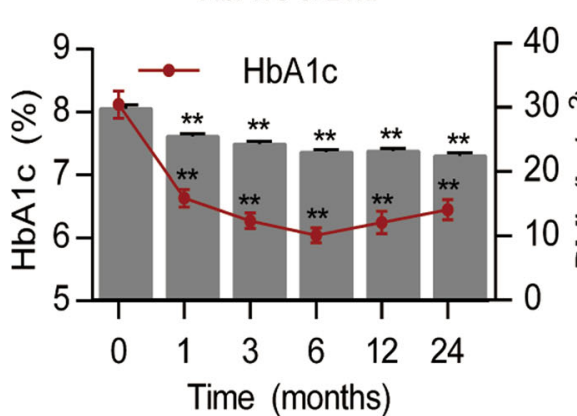

f

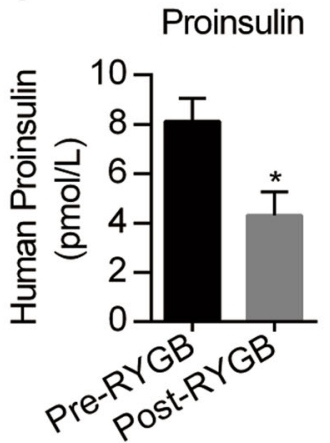

g

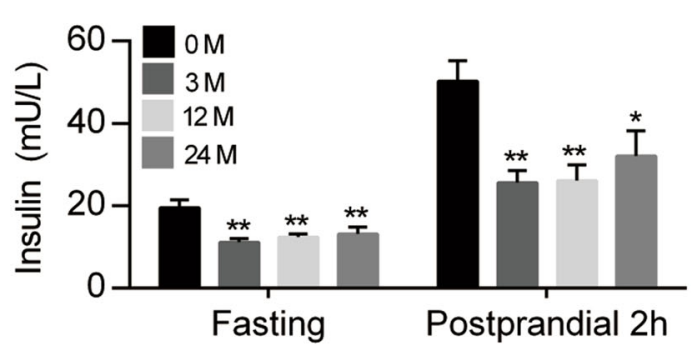

h

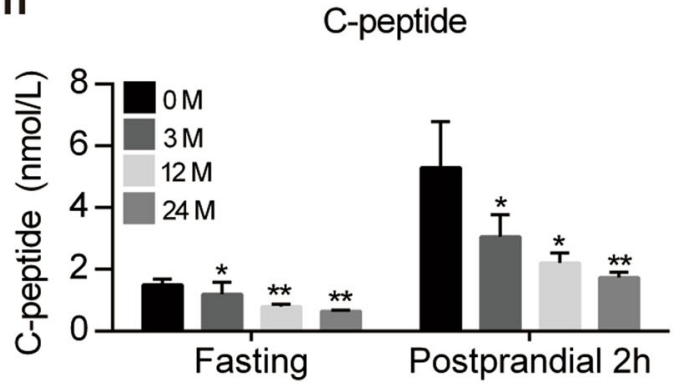

Fig. 7 RYGB remits hyperglycemia in patients with type $\mathbf{2}$ diabetes. $\mathbf{a}$, b 24-h dynamic and mean blood glucose values in patients pre- and postRYGB surgery ( $n=9$ pre-RYGB and 15 post-RYGB). c, d OGT results for patients at 0,3,12 and 24 months after surgery $(n=25-83$ ). e Hemoglobin A1c (HbA1c) levels and body mass index (BMI) at different time points for T2DM patients who underwent RYGB surgery $(n=23-87)$. f Serum proinsulin levels of patients before and after surgery $(n=9-12)$. $\mathbf{g}$ Plasma insulin levels and AUC values of the insulin release test at different time points for T2DM patients who underwent RYGB surgery (0 M (pre-RYGB); 3 M (3 months); 12 M (12 months); 24 M (24 months), $n=25-70)$. $\mathbf{h}$ Serum C-peptide levels of T2DM patients at the indicated times before and after RYGB upon glucose stimulation for $2 \mathrm{~h}(n=24-78)$. Values are shown as the mean \pm s.e.m. The significance was calculated using a two-tailed unpaired $t$-test (b), Mann-Whitney nonparametric $U$ test (f), and repeated-measures analysis $(\mathbf{d}, \mathbf{g}-\mathbf{h}) .{ }^{*} P<0.05$ and ${ }^{*} P<0.01$ compared with the pre-RYGB values

plasma proinsulin levels. However, the effect of RYGB on the structure and function of secretory granules in pancreatic islets worthy further investigates through electron microscope analysis. It is reported that insulin sensitivity also contributes to the maintenance of glucose homeostasis $^{36}$. Unexpectedly, RYGB decreased, not increased, insulin sensitivity in this study. The following evidence supports this point: 1 . the hyperinsulinemic-euglycemic clamp results showed a decrease in insulin sensitivity in conscious rats; 2. phosphorylation of the insulin receptor and flux through the PI3K/Akt signaling pathway were reduced in the liver, skeletal muscle and fat; 3. HGP was enhanced under both basal and hyperinsulinemiceuglycemic conditions, indicating weaker insulin action; and 4. decreased glucose uptake was observed in skeletal muscle and adipose tissue. From an evolutionary view, mammalian survival has adapted to insulin-independent circumstances because of food scarcity. In times of famine, starving people rarely suffer from obesity and type 2 diabetes despite insufficient energy intake diminishes 


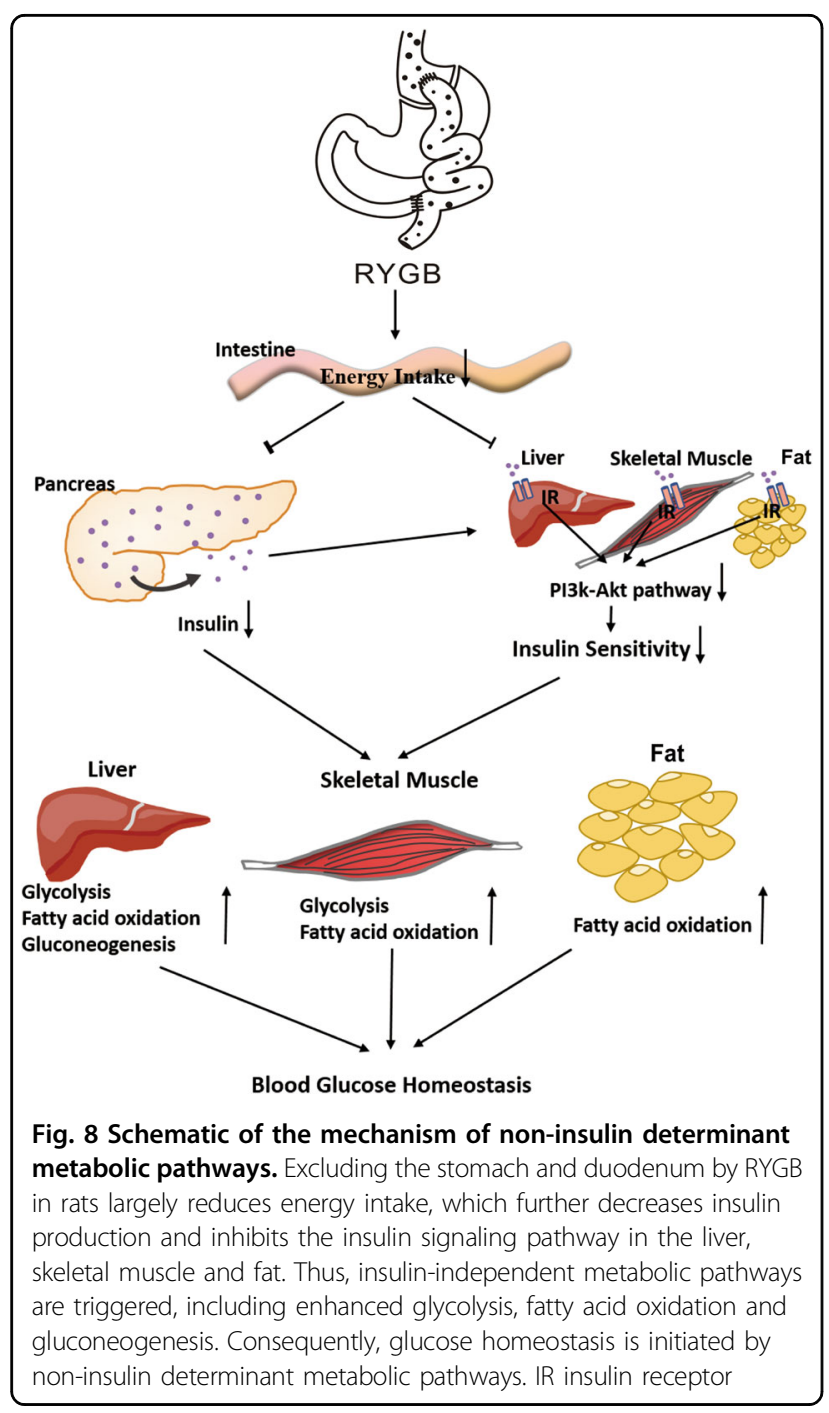

insulin production and the energy expenditure to avert hypoglycemia ${ }^{5,37}$. In fact, the effect of RYGB somewhat resembles a moderate famine state by placing a compulsory limit on gut energy intake. Taken together, our results demonstrate that the effects of RYGB on glucose homeostasis are independent of insulin action. Furthermore, these effects can be validated in rodents and patients with diabetes regardless of their islet status.

The next critical question is how glucose homeostasis is maintained under insulin deficiency and insensitivity. Although muscle and adipose tissue are insulin insensitive and uptake less glucose in rats after RYGB, glucose uptake is enhanced in brain and jejunum in this study. It is well known that glucose is a critical energy substrate required for brain functioning ${ }^{38}$, and the intestine exhibited the highest rate of glucose uptake and became a major tissue for glucose disposal after RYGB ${ }^{11}$. Thus, the decreased glucose absorption in metabolic organs, but enhanced glucose utilization of vital organs might contribute to the maintenance of glucose homeostasis after RYGB.

The binding of insulin to its receptors activates the canonical insulin receptor substrate/PI3K pathway, which potently inhibits both glycogenolysis and gluconeogenesis $^{2}$. Gluconeogenesis is catalyzed by several key enzymes, such PEPCK and G6Pase ${ }^{24,25}$. Glycolysis is catalyzed by PFK, PK, and HK, among others ${ }^{39}$. We showed that RYGB markedly increased the expression of these key enzymes in the rat liver, skeletal muscle and adipose tissue. In addition, this study clearly showed a shift in energy substrate usage from glucose to fatty acid metabolism after surgery. RYGB enhanced fatty acid degradation and promoted fatty acid utilization. These alterations are involved in changes in ACC, CPT1. Importantly, RYGB also impacted a plethora of crucial signaling molecules that regulate glucose and fatty acid metabolism, such as AMPK and PPARs. Some of these molecules are targets of hypoglycemic drugs, such as the AMPK activator metformin, GLP-1 agonists, adiponectin, and PPAR activators such as those in the glitazone family ${ }^{40,41}$. Although incretin hormones play an important role for glucose homeostasis, and plasma GLP-1 level elevated after RYGB in this study, previous study shows that GLP-1 is not the cause of the amelioration of hyperglycemia after RYGB ${ }^{42}$. Thus, no single drug or drug combination has been able to completely normalize blood glucose, as compared with RYGB $^{9,32,43}$. Our study indicates that a complex metabolic network, not a single target, is triggered by RYGB, which is responsible for the regulation of glucose homeostasis.

It was astonishing that RYGB led to not only weight loss but also glucose control and end-organ health ${ }^{9}$. Clinical studies have demonstrated that metabolic surgery also remits cardiometabolic complications, dementia, and cancer beyond the remission of hyperglycemia ${ }^{8,9,44}$. Hyperinsulinemia is regarded as the root cause of these noncommunicable diseases ${ }^{7,45}$. In modern society, excessive energy uptake and lower energy expenditure lead to hyperinsulinemia and fat accumulation in humans ${ }^{5,46}$. Persistent hyperinsulinemia leads to cellular and target organ dysfunction ${ }^{46,47}$. Therefore, caloric restriction and physical exercise are essential to maintain energy balance by antagonizing the action of hyperinsulinemia $^{48-50}$. Although lifestyle changes remarkably improve insulin sensitivity and glucose homeostasis, these behavioral interventions suffer from poor compliance and are insufficient to overcome overt obesity and diabetes ${ }^{48,49}$. These findings suggest that the principle mechanisms of RYGB are distinguished from those of lifestyle changes. In addition, our microarray chip analysis showed that RYGB impacted genes involved in inflammation, oxidative stress, cell growth, immunity, etc. These factors are closely associated with the development of cardiometabolic diseases, cancer, and brain disorders ${ }^{51}$. 
In summary, a strong non-insulin-dependent mechanism maintains glucose homeostasis and energy balance in physiological states. Disrupting this mechanism likely leads to disorders characterized by energy metabolism dysregulation. Based on the mechanism of action of RYGB, the development of a noninvasive intervention involving the gastrointestinal tract is a promising therapeutic route to combat these disorders.

\section{Materials and methods}

\section{Animals and rodent diabetes model}

The animal experiments and animal care were performed in compliance with and were approved by the ethics committee of the third affiliated hospital to the Third Military Medical University. Non-obese male Sprague Dawley (SD) rats, C57BL/6 J mice were used in this study. Rats and mice were housed under a 12-hour day-night cycle and had free access to food and water. Rats were randomly assigned to the sham-treated or metabolic surgery groups. The protocol is provided in the text. After an overnight fast, SD rats were intraperitoneally injected with vehicle $(0.1 \mathrm{M}$ sodium citrate, $\mathrm{pH}$ 4.5) or $45 \mathrm{mg} / \mathrm{kg} \mathrm{STZ} \mathrm{(Sigma-Aldrich} \mathrm{Co} \mathrm{LLC,} \mathrm{St.} \mathrm{Louis,}$ $\mathrm{MO}$ ) according to the protocol from the Animal Models of Diabetes Complications Consortium (AMDCC). After three days, rats were described as diabetic if their fasting blood glucose levels were greater than $300 \mathrm{mg} / \mathrm{dL}$ (16.7 $\mathrm{mmol} / \mathrm{L}$ ) for three consecutive days ${ }^{52-54}$.

\section{Oral glucose tolerance test (OGTT) and intraperitoneal glucose tolerance tests (IPGTT)}

Animals were fasted overnight before all the tests. After fasting, animals were given the glucose solution $(2 \mathrm{~g}$ glucose $/ \mathrm{kg}$ body weight) orally for OGTT, or glucose solution $(2 \mathrm{~g}$ glucose $/ \mathrm{kg}$ body weight or $4 \mathrm{~g}$ glucose $/ \mathrm{kg}$ body weight) injected intraperitoneally for IPGTT ${ }^{55,56}$. OGTT and IPGTT were performed in separate days. Blood glucose were monitored by the glucose telemetric transmitter before and after the test, or using the Nova StatStrip Xpress glucometer (Nova Biomedical, Waltham, MA) by cutting the tail and gently massaging blood onto a glucose test strip.

\section{Surgery and post-surgery procedures}

After an overnight fast, animals were deeply anesthetized with isoflurane (4\% induction and $1.5 \%$ maintenance; VIP-3000 Vaporizer; Matrix, Orchard Park, NY), and the abdominal organs were exposed by laparotomy. Animals were fasted for $12 \mathrm{~h}$ after surgery. After evaluating the health status and behavior of each animal, a limited liquid diet was reintroduced $12-72 \mathrm{~h}$ after the operation. Three days after surgery, normal chow was provided ad libitum to the animals ${ }^{11,44}$. Animals used in this study underwent operations in different cohorts, and STZ was injected 2 weeks before surgery ${ }^{57}$.

\section{Rat RYGB}

The total length of the small intestine was measured, the ligament of Treitz was identified, and the small intestine was divided at the appropriate distance $(8-10 \mathrm{~cm})$ downstream of this ligament ${ }^{11}$. A gastrojejunostomy and an end-to-side jejuno-jejunostomy $(18-20 \mathrm{~cm}$ from the ligament of Treitz) were created with a 6-0 silk interrupted suture ${ }^{57}$. A gastric pouch consisting of $10 \%$ of the total stomach volume was created using scissors, and the distal cut end of the stomach was sutured with 6-0 silk. The intestine remained hydrated during this procedure. The gastric artery was preserved, and only the small vascular branches were cauterized with electrocoagulation. The laparotomy was closed with a 4-0 Prolene suture in two layers.

\section{Mouse RYGB}

The upper gastrointestinal tract was exposed, and the length of the small intestine was measured. The intestine was cut at a distance of $10-15 \%$ of the intestinal length from the pylorus. The stomach was gently dissected from the spleen and liver by using cotton swabs, and the gastroesophageal junction was fully externalized. The left gastric vessels and vagal fibers were removed to create a small pouch (approximately $2 \%$ of the stomach capacity). The stomach remnant was closed using a continuous stitch with an 8-0 Prolene suture (Ethicon US, LLC, USA). The stomach pouch was then anastomosed with a distal jejunum, and a jejuno-jejunostomy was performed using an interrupted stitch with an 8-0 Prolene suture at a distance of $10-15 \%$ of the intestinal length from the gastrojejunostomy. Warm saline was regularly added to wet the tissue. The abdominal muscle and skin were closed using an interrupted stitch with a 6-0 silk suture ${ }^{26}$.

\section{Vertical sleeve gastrectomy (VSG)}

The stomach was isolated outside the abdominal cavity and placed on warm saline-soaked gauze pads. It was then freed from the spleen and liver, and the lateral $80 \%$ of the stomach was cut, leaving a tubular gastric remnant (closed using an interrupted stitch with a 6-0 silk suture) while keeping the proximal and distal ends intact. Finally, the abdominal wall was closed in layers using an interrupted stitch with a $4-0$ silk suture ${ }^{26}$.

\section{Duodenal-jejunal bypass (DJB)}

The stomach was fully externalized with a VSG surgical procedure, and the jejunum was transected using the previously described rat RYGB procedure. The pyloric sphincter was ligated with a 4-0 silk suture. An incision 
was created on the lateral wall of the stomach, and the distal jejunum was anastomosed to the stomach. A jejunojejunostomy was performed with an RYGB procedure. The abdominal wall was closed in layers using an interrupted stitch with a 4-0 silk suture ${ }^{10}$.

\section{Restoration of the gastroduodenal passage after RYGB surgery}

A loop of the jejunum $(1 \mathrm{~cm})$ was isolated and transposed between the oesophagus and the stomach to restore the gastroduodenal passage of nutrients after RYGB surgery $^{58}$. After the operation, nutrients flowed from the esophagus through the jejunal loop to the stomach in a latero-terminal fashion involving the duodenum, jejunum, and remaining intestinal tract. Anastomoses were performed with a 6-0 silk interrupted suture ${ }^{11}$.

\section{Sham operation}

In all experiments, animals undergoing a sham operation were used as controls. The sham operation was consisted of laparotomy, jejunal transection, and sutured; and was performed in animals that were matched (ageand weight-) to rats underwent RYGB or restoration.

\section{Continuous blood glucose monitoring in rats}

Glucose telemetric transmitters (HD-XG transmitter; Data Sciences International, Saint Paul, MN) were surgically implanted in SD rats after metabolic surgery and before the STZ injection, according to a previously reported protocol ${ }^{18,59}$. The catheter portion of the implant was placed into the distal portion of the descending aorta. After rats recovered from surgery for several days, the blood glucose levels in conscious and unrestrained rats were measured by telemetry until the end of the study. We collected data for $10 \mathrm{~s}$ every minute and used the mean values for the statistical analysis.

\section{Hyperinsulinemic-euglycaemic clamp}

The test was performed as previously described, and insulin sensitivity was represented by the amount of glucose infused during the last $60 \mathrm{~min}$ of clamping. In detail, two catheters were separately introduced into the carotid artery and right jugular vein. Briefly, a primed continuous infusion of HPLC-purified [3-H3] glucose (Amersham, Los Angeles, CA; $6 \mathrm{mCi}$ bolus, $0.2 \mathrm{mCi} / \mathrm{min}$ ) through the carotid artery catheter was initiated at $0 \mathrm{~min}$ and maintained throughout the study. The hyperinsulinemiceuglycemic clamp was performed, insulin $(6 \mathrm{mU} / \mathrm{kg} /$ $\mathrm{min}$ ) was continuously infused, and a variable infusion of $25 \%$ glucose was started and adjusted every $5 \mathrm{~min}$ to maintain the plasma glucose concentration at $6 \mathrm{mmol}$. $\mathrm{GIR}=\mathrm{Y} \times 25 /(6 \times \mathrm{W})(\mathrm{Y}$ : glucose infusion rate, $\mathrm{ml} / \mathrm{h} ; \mathrm{W}$ : body weight, $\mathrm{kg}$ ). Blood samples were collected from the jugular vein catheter at $0,120,200,220,230$, and $240 \mathrm{~min}$ and used to determine insulin levels. To ascertain insulinmediated glucose uptake in individual tissues, 2-deoxy-D[H3] glucose (2-DG) (Amersham; $30 \mathrm{mCi}$ bolus) was administered $45 \mathrm{~min}$ before the end of the clamp studies. Extra blood samples $(100 \mu \mathrm{L})$ were taken at 2, 5, 10, 15, 20, 30 and $45 \mathrm{~min}$ after the infusion to determine tracer disappearance. At the end of the clamp, the rats were anesthetized, and tissue samples were frozen in liquid nitrogen and stored at $-80^{\circ} \mathrm{C}$ for subsequent analysis. Insulin sensitivity was computed with previously described formulas ${ }^{60}$.

\section{Isolation of skeletal muscle and liver mitochondria}

The rats were sacrificed, and their musculi soleus tissues were promptly removed, washed with phosphate-buffered saline, and weighed. The tendon was removed. Each musculi soleus was placed in ice-cold buffer $(180 \mathrm{mM}$ $\mathrm{KCl}, 10 \mathrm{mM}$ Tris, and $0.5 \mathrm{mM} \mathrm{Na} \mathrm{N}_{2}$ EDTA, $\left.\mathrm{pH} 7.4\right)$, cut into tiny pieces, and further homogenized in buffer containing $180 \mathrm{mM} \mathrm{KCl}, 10 \mathrm{mM}$ Tris, $0.5 \mathrm{mM} \mathrm{Na}_{2}$ EDTA, and $1 \mathrm{~g} / \mathrm{L}$ BSA, pH 7.4, by using a Dounce homogenizer. Muscle mitochondria were isolated by differential centrifugation. Mitochondria were weighed and suspended in MiR06 solution. The remaining sample was stored at -80 ${ }^{\circ} \mathrm{C}$ for subsequent analysis ${ }^{61}$.

\section{High-resolution respirometry}

The mitochondrial respiration rate was measured by using an Oxygraph-2k (Oroboros, Innsbruck, Austria) with two chambers at $37^{\circ} \mathrm{C}$, and $1.0 \mathrm{mg}$ of mitochondria was added into each high-resolution respirometry chamber containing $2 \mathrm{~mL}$ of MiR06 solution. The Datlab software package (Oroboros Instruments) was used for data acquisition and analysis. The substrate-uncoupler-inhibitor titration (SUIT) reference protocol used to evaluate mitochondrial function included ADP $(450 \mu \mathrm{M})$, Mitochondria (Mt), Oct $(0.5 \mathrm{mM})$, Malate $(0.05 \mathrm{mM}, 0.1 \mathrm{mM}$, and $2 \mathrm{mM})$, cytochrome c $(10 \mu \mathrm{M})$, a titration of the uncoupler FCCP (injected stepwise up to $1-1.5 \mu \mathrm{M}$ ), rotenone $(0.5 \mu \mathrm{M})$, and myxothiazol $(0.5 \mu \mathrm{M})$. The ADP was added before Mt to accelerate the depletion of endogenous substrates. Then, the fatty acid oxidation (FAO) rate was determined by using Oct and Malate. Cytochrome $\mathrm{c}$ was used to confirm the integrity of the outer mitochondrial membrane. FCCP was titrated to evaluate the maximal electron transfer system (ETS) capacity. Residual oxygen consumption (ROX) was determined by adding rotenone and myxothiazol.

\section{Energy expenditure and Respiratory Exchange Ratio}

To investigate the effect of RYGB surgery on mouse energy expenditure (EE) and respiratory exchange ratio (RER), the Comprehensive Laboratory Animal 
Monitoring System (CLAMS; Columbus Instruments, $\mathrm{OH}, \mathrm{USA}$ ) was used to monitor the oxygen consumption and respiratory exchange ratio (RER) of mice. Mice were weighted and then housed individually in monitoring cages for $48 \mathrm{~h}$ and the room temperature was maintained in $22^{\circ} \mathrm{C}$ under a 12 -hour/12-hour day/night cycle. Mice were acclimatized to the cage for $24 \mathrm{~h}$ before each trial. Data was analyzed by the suited Oxymax software package (Columbus Instruments). The contents of oxygen $(\mathrm{O} 2)$ and carbon dioxide $(\mathrm{CO} 2)$ in the air sampled from the cage was determined by the separated sensor (Columbus Instruments). Carbon dioxide output (VCO2) and oxygen uptake (VO2) was counted between each interval. Then RER was calculated according to the equation $\mathrm{RER}=\mathrm{VCO} 2 / \mathrm{VO} 2$. Heat production was calculated according to the equation: $\mathrm{EE}=(3.815+1.232 \times$ $\mathrm{RER}) \times V \mathrm{O} 2 \times 0.001(\mathrm{kcal} /[\mathrm{kg} \times \mathrm{h}])^{62}$.

\section{Metabolic assays and ELISA kits}

For insulin, glucagon, DPP-4, Ghrelin, Leptin, Gastrin, and FGF-21 assays, blood was collected into heparinized tubes on ice. For GIP and GLP-1(7-36) assays, blood was collected into tubes, on ice, containing EDTA and dipeptidyl peptidase IV (DPP-IV) inhibitor (Millipore, Billerica, MA, USA $)^{10}$. Plasma was obtained by centrifugation of heparinized blood at $4{ }^{\circ} \mathrm{C}$ for $20 \mathrm{~min}$ at $1,200 \mathrm{~g}$ and subjected to enzyme immunoassays. The plasma glucagon, GLP-1 (7-36), GIP, Ghrelin, Leptin (Millipore, Billerica, MA, USA) and DPP-4 Gastrin, and FGF-21 assays were performed using commercialized kits according to manufacturer's instructions. The plasma insulin levels were determined by ELISA kits and radioimmunoassay (ELISA, Mercodia, Uppsala, Sweden and RIA, Millipore, Billerica, MA, USA), respectively. All the samples were performed in bipartites.GLP-1 levels were measured $30 \mathrm{~min}$ after a meal of glucose solution ( $2 \mathrm{~g} / \mathrm{kg}$ body weight).

\section{Fecal analysis}

24-hours fecal pellets from individual rat were collected to have sufficient material for analysis. Fecal total protein concentrations were quantified using Kjeldahl method ${ }^{63}$, total fat were extracted and measured by the procedure of Soxhlet extraction. Total carbohydrate and totalcalories were estimated as follows:

Total carbohydrate $(\%)=100-($ Ash + Moisture + Protein + Fat) \%

Total calories $(\mathrm{kj})=$ Carbohydrate $\times 17+$ Protein $\times$ $17+$ Fat $\times 37$

Results were then calibrated with the weight of the sample.

\section{Histology and Immunofluorescent staining}

Whole pancreas was embedded in Tissue Tek (Sakura Fneteci), frozen, cut into sections (8 um). Histology slides containing sections from the pancreas were fixed with $4 \%$ paraformaldehyde, permeabilized with $0.01 \%$ Triton $\mathrm{X}$-100.For histology, the slides from each group were stained with hematoxylin and eosin. For Immunofluorescent staining, nonspecific binding sites were blocked in $10 \%$ bovine serum in PBS at room temperature for $30 \mathrm{~min}$. The slides were then incubated at $4{ }^{\circ} \mathrm{C}$ overnight with diluted primary antibodies and with Alexa Fluor labeled secondary antibodies at $37^{\circ} \mathrm{C}$ for $60 \mathrm{mins}$ in the dark followed by DAPI for 30 mins at room temperature in the dark. The slides were then rinsed with PBS and evaluated under a fluorescence microscope. The primary antibodies used were anti-insulin antibody (ab6995, Abcam), anti-glucagon antibody (ab8055, Abcam). The images were collected using a Nikon TE2000-U inverted fluorescence microscope (Nikon Co., Tokyo, Japan).

\section{Western blot}

Protein was extracted from the frozen tissues of animals, including islet, liver, skeletal muscle and white adipose tissue. Protein concentrations were determined by BCA method. Equal amounts of protein $(20 \mu \mathrm{g}$ per lane) were separated by SDS-PAGE and transferred onto polyvinylidene difluoride membranes (Millipore). After blocking, the filters were incubated with the primary antibodies. Antibodies including, Akt, p-Akt, InsR (insulin receptor), p-InsR, Acc, p-Acc, HK2 (hexokinase 2), PFKL (6-phosphofructokinase, liver type), PKM1/2 (pyruvate kinase muscle isozyme), PDH (pyruvate dehydrogenase), PDHK1 (pyruvate dehydrogenase kinase isozyme 1) were purchased from Cell Signaling Technology. Antibodies, including PKLR (pyruvate kinase, liver and RBC), PEPCK (phosphoenolpyruvate carboxykinase), CPT1, PTBP1 were purchased from Abcam. Antibodies including GCK (glucokinase), PFK1 (phosphofructokinase 1), G6Pase (glucose-6-Phosphatase), PPAR $\alpha$, PPAR $\delta$ PPAR $\gamma$ were purchased from Santa cruz biotechnology. After washes and incubation with the appropriate horseradish peroxidase-conjugated secondary antibody (Santa Cruz Biotechnology), the immune complexes were visualized using a chemiluminescence reagent. Western blot results were densitometrically quantified with Quantity One software (Bio-Rad), and the intensity values were normalized to $\beta$-actin.

\section{Gene expression microarrays and data analysis}

Total RNA was extracted from the frozen tissues by using TRIzol (Invitrogen). The RNA quantity and quality were measured with a NanoDrop ND-1000. RNA integrity was assessed by using standard denaturing agarose gel electrophoresis. The Rat $4 \times 44 \mathrm{~K}$ Gene Expression Array was manufactured by Agilent. The updated content provided full coverage of rat genes and transcripts. Sample 
labeling and array hybridization were performed according to the Agilent One-Color Microarray-Based Gene Expression Analysis protocol (Agilent Technology). Briefly, the total RNA from each sample was linearly amplified and labeled with Cy3-UTP. The labeled cRNAs were purified with an RNeasy Mini Kit (Qiagen). The concentration and specific activity of the labeled cRNAs (pmol Cy3/ $\mu \mathrm{g}$ cRNA) were measured with a NanoDrop ND-1000. Each labeled cRNA $(1 \mu \mathrm{g})$ was fragmented in $11 \mu \mathrm{l}$ of $10 \times$ Blocking Agent and $2.2 \mu \mathrm{l}$ of $25 \times$ Fragmentation Buffer. Then, the mixture was heated at $60^{\circ} \mathrm{C}$ for 30 min. Finally, $55 \mu \mathrm{l}$ of $2 \times$ GE Hybridization buffer was added to dilute the labeled cRNA. One hundred microliters of hybridization solution was dispensed into the gasket slide and assembled to create the gene expression microarray slide. The slides were incubated for $17 \mathrm{~h}$ at $65^{\circ} \mathrm{C}$ in an Agilent Hybridization Oven. The hybridized arrays were washed, fixed and scanned with the Agilent DNA Microarray Scanner (part number G2505C). Agilent Feature Extraction software (version 11.0.1.1) was used to analyse the acquired array images. Quantile normalization and subsequent data processing were performed using the Gene Spring GX v12.1 software package (Agilent Technologies). After quantile normalization of the raw data, genes for which at least 46 out of 92 samples had Detected ("All Targets Value") flags were chosen for further analysis. Differentially expressed genes with a statistically significant difference between the two groups were identified through Volcano Plot filtering. Differentially expressed genes between the two samples were identified through Fold Change filtering. GO and KEGG Pathway analyses were performed in the standard enrichment computation method.

\section{Real-time PCR}

Total RNA from pancreas was isolated using TRIzol reagent (Invitrogen) according to the manufacturer's protocol. First strand cDNA was synthesized using random primers and M-MuLV Reverse Transcriptase (New England Biolabs). PCR reactions were carried out with the manufacturer (Light Cycler 96, Roche), using the Quanti Tect SYBR Green RT-PCR Kit (QIAGEN). The following primers were used: Ins1, 5'-AGGACCCGCAAGTGCCA CAA-3' (sense) and 5'-TCCACAATGCCACGCTTCT GC-3' (antisense); Ptbp1, 5' -AGTTCAAAGGTGATAACAGGAGCAC-3' (sense) and 5'-CATGTAGGCCATG AGGTCCACCAC-3' (antisense). The fluorescence curves were analyzed using LightCycler 96 software (Version 1.1).

\section{Clinical study}

The study cohort consisted of 87 type 2 diabetic patients and included 38 women and 49 men who met the criteria based on the 'Metabolic Surgery in the Treatment
Algorithm for Type 2 Diabetes: A Joint Statement by International Diabetes Organizations ${ }^{6}$. Patients were admitted and undergone routine physical examinations and systematic biochemical analyses before the surgery and were then followed up over two years. The examinations before and after surgery were performed. All patients undergone laparoscopic RYGB surgery at the Department of Surgery of the third affiliated hospital to the Third Military Medical University. All patients provided written informed consent. This study was approved by the ethics committee of the third affiliated hospital to the Third Military Medical University. The patients undergone oral glucose tolerance test (OGTT) to evaluate pancreatic $\beta$-cell function. OGTT (75-g glucose load, $2 \mathrm{~h}$ ) were performed before surgery and follow-up over two years. All patients simultaneously undergone a complete evaluation of pancreas islet function using insulin and Cpeptide release tests; plasma samples were collected at 0 , 30,60 , and $120 \mathrm{~min}$, and then assayed with a validated, highly sensitivity assay using a turbidimetric immunoassay (Orion Diagnostica, Espoo, Finland). The blood glucose concentrations were assayed using the glucose oxidase method $^{64}$.

\section{Statistical analyses}

All data were expressed as mean \pm s.e.m. and $P$ values were calculated using two-tailed Student's $t$-test for pairwise comparisons, one-way ANOVA for multiple comparisons, and two-way ANOVA for multiple comparisons involving two independent variables. ANOVA analyses were subjected to Bonferroni's post hoc test. Mann-Whitney non-parametric $U$ test was used to analyse data in abnormal distribution. Correlation analysis was performed by Linear Regression Univariate Test. $P<0.05$ was regarded as a significant effect or betweengroup difference. All tests were 2-tailed, and analyses were performed using either GraphPad Prism (GraphPad Software Inc., La Jolla, CA) or SPSS (version 12.0; SPSS, Inc., Chicago, IL).

\section{Acknowledgements \\ This work was supported by grants from the National Natural Science Foundation of China (81721001, 31430042, and 81400351) and Innovative Research Team in University (IRT1216). We thank Lijuan Wang, Changyuan Meng, Shiqiang Xiong, Shaoyang Lin, Xing Wei and Yuanting Cui for their technical assistance.}

\section{Author details}

${ }^{1}$ Department of Hypertension and Endocrinology, Center for Hypertension and Metabolic Diseases, Daping Hospital, Third Military Medical University, Chongqing Institute of Hypertension, Chongqing 400042, China. ${ }^{2}$ Department of Gastrointestinal Metabolic Surgery, Daping Hospital, Third Military Medical University, Chongqing 400042, China. ${ }^{3}$ Department of Endocrinology, Xinqiao Hospital, Third Military Medical University, Chongqing 400037, China.

${ }^{4}$ Department of Endocrinology, the Second Affiliated Hospital, Chongqing Medical University, Chongqing 400010, China. ${ }^{5}$ Institute of Vascular Medicine and School of Biomedical Sciences, Chinese University of Hong Kong, BMSB315, Shatin, Hong Kong 00852, China 


\section{Author contributions}

Z.Z., Z.L., X.W. and F.S. designed the research and co-wrote the paper. Z.L., X.W., F.S., H.Z. and P.G. performed experiments. Y.P. and A. W. assisted with the animal work. F.S., Q.L., and X.Z. performed and analyzed clinical studies. J.C. W.T. and Q.L. assisted with the clinical work. Z.L., X.W., F.S., H.Z., P.G. and Q.L. performed statistical analyses. P.G., Z.Y., H.Z., G.Y., Y.H. and D.L. reviewed the manuscript and provided comments and experiments assistance. Z.Z. directed the research and co-wrote the paper, with assistance from all other authors. The authors declare no competing financial interests.

\section{Conflict of interest}

The authors declare that they have no conflict of interest.

\section{Publisher's note}

Springer Nature remains neutral with regard to jurisdictional claims in published maps and institutional affiliations.

Supplementary Information accompanies the paper at (https://doi.org/ 10.1038/s41421-018-0062-x).

Received: 15 May 2018 Revised: 13 August 2018 Accepted: 30 August 2018 Published online: 25 September 2018

\section{References}

1. Roth, J. et al. Insulin's discovery: new insights on its ninetieth birthday. Diabetes Metab. Res Rev. 28, 293-304 (2012).

2. Saltiel, A. R. \& Kahn, C. R. Insulin signalling and the regulation of glucose and lipid metabolism. Nature 414, 799-806 (2001).

3. Schwartz, M. W. et al. Cooperation between brain and islet in glucose homeostasis and diabetes. Nature 503, 59-66 (2013).

4. Kahn, S. E., Cooper, M. E. \& Del Prato, S. Pathophysiology and treatment of type 2 diabetes: perspectives on the past, present, and future. Lancet $\mathbf{3 8 3}$, 1068-1083 (2014).

5. Ashcroft, F. M. \& Rorsman, P. Diabetes mellitus and the beta cell: the last ten years. Cell 148, 1160-1171 (2012)

6. Rubino, F. et al. Metabolic surgery in the treatment algorithm for Type 2 diabetes: a Joint Statement by International Diabetes Organizations. Diabetes Care. 39, 861-877 (2016)

7. Pories, W. J. \& Dohm, G. L. Diabetes: have we got it all wrong? Hyperinsulinism as the culprit: surgery provides the evidence. Diabetes Care. 35, 2438-2442 (2012).

8. Sjostrom, L. et al. Association of bariatric surgery with long-term remission of type 2 diabetes and with microvascular and macrovascular complications. JAMA 311, 2297-2304 (2014)

9. Miras, A. D. \& le Roux, C. W. Metabolic Surgery in a Pill. Cell Metab. 25, 985-987 (2017).

10. Breen, D. M. et al. Jejunal nutrient sensing is required for duodenal-jejunal bypass surgery to rapidly lower glucose concentrations in uncontrolled diabetes. Nat. Med. 18, 950-955 (2012).

11. Saeidi, N. et al. Reprogramming of intestinal glucose metabolism and glycemic control in rats after gastric bypass. Science 341, 406-410 (2013).

12. Ryan, K. K. et al. FXR is a molecular target for the effects of vertical sleeve gastrectomy. Nature 509, 183-188 (2014)

13. Dixon, J. B., le Roux, C. W., Rubino, F. \& Zimmet, P. Bariatric surgery for type 2 diabetes. Lancet 379, 2300-2311 (2012)

14. Drucker, D. J. Deciphering metabolic messages from the gut drives therapeutic innovation: the 2014 Banting Lecture. Diabetes 64, 317-326 (2015).

15. Faucher, P. et al. Bariatric surgery in obese patients with type 1 diabetes: effects on weight loss and metabolic control. Obes. Surg. 26, 2370-2378 (2016).

16. Fujikawa, T., Chuang, J. C., Sakata, I., Ramadori, G. \& Coppari, R. Leptin therapy improves insulin-deficient type 1 diabetes by CNS-dependent mechanisms in mice. Proc. Natl. Acad. Sci. USA. 107, 17391-17396 (2010).

17. Kubota, T., Kubota, N. \& Kadowaki, T. Imbalanced insulin actions in obesity and type 2 diabetes: key mouse models of insulin signaling pathway. Cell Metab. 25, 797-810 (2017).
18. Brockway, R. et al. Fully implantable arterial blood glucose device for metabolic research applications in rats for two months. J. Diabetes Sci. Technol. 9 771-781 (2015).

19. Itoh, N. \& Okamoto, H. Translational control of proinsulin synthesis by glucose Nature 283, 100-102 (1980).

20. Wicksteed, B., Alarcon, C., Briaud, I., Lingohr, M. K. \& Rhodes, C. J. Glucoseinduced translational control of proinsulin biosynthesis is proportional to preproinsulin mRNA levels in islet beta-cells but not regulated via a positive feedback of secreted insulin. J. Biol. Chem. 278, 42080-42090 (2003).

21. Knoch, K. P. et al. Polypyrimidine tract-binding protein promotes insulin secretory granule biogenesis. Nat. Cell Biol. 6, 207-214 (2004).

22. Edgerton, D. S. et al. Effects of insulin on the metabolic control of hepatic gluconeogenesis in vivo. Diabetes 58, 2766-2775 (2009).

23. Lin, H. V. \& Accili, D. Hormonal regulation of hepatic glucose production in health and disease. Cell Metab. 14, 9-19 (2011).

24. Yoon, J. C. et al. Control of hepatic gluconeogenesis through the transcriptional coactivator PGC-1. Nature 413, 131-138 (2001).

25. Yabaluri, N. \& Bashyam, M. D. Hormonal regulation of gluconeogenic gene transcription in the liver. J. Biosci. 35, 473-484 (2010).

26. Bruinsma, B. G., Uygun, K., Yarmush, M. L. \& Saeidi, N. Surgical models of Rouxen- $Y$ gastric bypass surgery and sleeve gastrectomy in rats and mice. Nat. Protoc. 10, 495-507 (2015).

27. Duca, F. A., Bauer, P. V., Hamr, S. C. \& Lam, T. K. Glucoregulatory relevance of small intestinal nutrient sensing in physiology, bariatric surgery, and pharmacology. Cell Metab. 22, 367-380 (2015).

28. Zietek, T. \& Daniel, H. Intestinal nutrient sensing and blood glucose control. Curr. Opin. Clin. Nutr. Metab. Care. 18, 381-388 (2015).

29. Fan, W. et al. PPARdelta promotes running endurance by preserving glucose. Cell Metab. 25, 1186-1193 e1184 (2017).

30. Kirwan, J. P. et al. Bariatric surgery in obese patients with type 1 diabetes Diabetes Care. 39, 941-948 (2016).

31. Ikramuddin, S. et al. Roux-en-Y gastric bypass for diabetes (the Diabetes Surgery Study): 2-year outcomes of a 5-year, randomised, controlled trial. Lancet Diabetes \& Endocrinol. 3, 413-422 (2015).

32. Schauer, P. R. et al. Bariatric surgery versus intensive medical therapy in obese patients with diabetes. N. Engl. J. Med. 366, 1567-1576 (2012).

33. Camastra, S. et al. Long-term effects of bariatric surgery on meal disposal and beta-cell function in diabetic and nondiabetic patients. Diabetes $\mathbf{6 2}$ 3709-3717 (2013)

34. Magkos, F., Fabbrini, E., Patterson, B. W., Eagon, J. C. \& Klein, S. Portal vein and systemic adiponectin concentrations are closely linked with hepatic glucose and lipoprotein kinetics in extremely obese subjects. Metabolism 60, 1641-1648 (2011)

35. Umpleby A M Scobie I N Boroujerdi, M A \& Sonksen, P. H. The effect of starvation on leucine, alanine and glucose metabolism in obese subjects. Eur. J. Clin. Invest. 25, 619-626 (1995).

36. Bradley, D. et al. Gastric bypass and banding equally improve insulin sensitivity and beta cell function. J. Clin. Invest. 122, 4667-4674 (2012).

37. Juhl, $\mathrm{C}$. et al. Effects of fasting on physiologically pulsatile insulin release in healthy humans. Diabetes 51(Suppl 1), S255-S257 (2002).

38. Rehni, A. K. \& Dave, K. R. Impact of hypoglycemia on brain metabolism during diabetes. Mol. Neurobiol. https://doi.org/10.1007/s12035-018-1044-6 (2018).

39. Hagiwara, A. et al. Hepatic mTORC2 activates glycolysis and lipogenesis through Akt, glucokinase, and SREBP1c. Cell Metab. 15, 725-738 (2012).

40. Gastaldi, G. et al. Upregulation of peroxisome proliferator-activated receptor gamma coactivator gene (PGC1A) during weight loss is related to insulin sensitivity but not to energy expenditure. Diabetologia 50, 2348-2355 (2007)

41. $\mathrm{Xu}, \mathrm{X}$. J. et al. Improved insulin sensitivity 3 months after RYGB surgery is associated with increased subcutaneous adipose tissue AMPK activity and decreased oxidative stress. Diabetes 64, 3155-3159 (2015).

42. Vidal, J., de Hollanda, A. \& Jimenez, A. GLP-1 is not the key mediator of the health benefits of metabolic surgery. Surg. Obes. Relat. Dis. 12, 1225-1229 (2016).

43. Mingrone, G. et al. Bariatric-metabolic surgery versus conventional medical treatment in obese patients with type 2 diabetes: 5 year follow-up of an openlabel, single-centre, randomised controlled trial. Lancet 386, 964-973 (2015).

44. Zhang, H. et al. Gastrointestinal intervention ameliorates high blood pressure through antagonizing overdrive of the sympathetic nerve in hypertensive patients and rats. J. Am. Heart Assoc. 3:e000929. https://doi.org/10.1161/ JAHA.114.000929 (2014) 
45. Corkey, B. E. Banting lecture 2011: hyperinsulinemia: cause or consequence? Diabetes 61, 4-13 (2012).

46. Kelly, C. T. et al. Hyperinsulinemic syndrome: the metabolic syndrome is broader than you think. Surgery 156, 405-411 (2014).

47. Weyer, C., Hanson, R. L., Tataranni, P. A., Bogardus, C. \& Pratley, R. E. A high fasting plasma insulin concentration predicts type 2 diabetes independent of insulin resistance: evidence for a pathogenic role of relative hyperinsulinemia. Diabetes 49, 2094-2101 (2000).

48. Castorena, C. M., Arias, E. B., Sharma, N. \& Cartee, G. D. Postexercise improvement in insulin-stimulated glucose uptake occurs concomitant with greater AS160 phosphorylation in muscle from normal and insulin-resistant rats. Diabetes 63, 2297-2308 (2014).

49. Laferrere, B. et al. Effect of weight loss by gastric bypass surgery versus hypocaloric diet on glucose and incretin levels in patients with type 2 diabetes. J. Clin. Endocrinol. Metab. 93, 2479-2485 (2008).

50. Schenk, S. et al. Sirt1 enhances skeletal muscle insulin sensitivity in mice during caloric restriction. J. Clin. Invest. 121, 4281-4288 (2011).

51. Verdile, G. et al. Inflammation and oxidative stress: the molecular connectivity between insulin resistance, obesity, and Alzheimer's disease. Mediat. Inflamm. 2015, 105828 (2015).

52. King, A. \& Bowe, J. Animal models for diabetes: understanding the pathogenesis and finding new treatments. Biochem Pharmacol. 99, 1-10 (2016).

53. King, A. J. F. The use of animal models in diabetes research. Br. J. Pharmacol. 166, 877-894 (2012)

54. Furman, B. L. Streptozotocin-induced diabetic models in mice and rats. Curr. Protoc. Pharmacol. 70, 547 41-20 (2015).

55. Wang, P. et al. Transient receptor potential vanilloid 1 activation enhances gut glucagon-like peptide-1 secretion and improves glucose homeostasis. Diabetes 61, 2155-2165 (2012).
56. Zhao, Y. et al. Sodium intake regulates glucose homeostasis through the PPARdelta/adiponectin-mediated SGLT2 pathway. Cell Metab. 23, 699-711 (2016).

57. Shin, A. C., Zheng, H. Y., Townsend, R. L., Sigalet, D. L. \& Berthoud, H. R. Mealinduced hormone responses in a rat model of Roux-en- $Y$ gastric bypass surgery. Endocrinology 151, 1588-1597 (2010).

58. Rubino, F. et al. The mechanism of diabetes control after gastrointestinal bypass surgery reveals a role of the proximal small intestine in the pathophysiology of type 2 diabetes. Ann. Surg. 244, 741-749 (2006).

59. Yang, D. et al. Activation of TRPV1 by dietary capsaicin improves endotheliumdependent vasorelaxation and prevents hypertension. Cell Metab. 12, 130-141 (2010).

60. Shiota, M. Measurement of glucose homeostasis in vivo: combination of tracers and clamp techniques. Methods Mol. Biol. 933, 229-253 (2012).

61. Xiong, S. et al. Ameliorating endothelial mitochondrial dysfunction restores coronary function via transient receptor potential vanilloid 1mediated protein kinase Avuncoupling protein 2 pathway. Hypertension 67, 451-460 (2016)

62. Lang, $H$. et al. Activation of TRPV1 attenuates high salt-induced cardiac hypertrophy through improvement of mitochondrial function. Br. J. Pharmacol. 172, 5548-5558 (2015).

63. Nozawa, S., Hakoda, A., Sakaida, K., Suzuki, T. \& Yasui, A. Method performance study of the determination of total nitrogen in soy sauce by the Kjeldahl method. Anal. Sci. 21, 1129-1132 (2005).

64. $\mathrm{He}, \mathrm{H}$. et al. Metformin-based treatment for obesity-related hypertension: a randomized, double-blind, placebo-controlled trial. J. Hypertens. 30, 1430-1439 (2012). 